\title{
Characterization of Silicon Quantum Dots' Properties and Kidney Toxicity in Mice ${ }^{\dagger}$
}

\author{
Roxana Elena Cristian 1, Miruna Silvia Stan 1,2 and Anca Dinischiotu 1,* \\ 1 Department of Biochemistry and Molecular Biology, Faculty of Biology, 91-95 Splaiul Independentei, \\ University of Bucharest, 050095 Bucharest, Romania; roxana.cristian@drd.unibuc.ro (R.E.C.); \\ miruna_stan@yahoo.com (M.S.S.) \\ 2 Department of Science and Engineering of Oxide Materials and Nanomaterials, Faculty of Applied \\ Chemistry and Materials Science, University Politehnica of Bucharest, 1-7 Polizu Street, \\ 011061 Bucharest, Romania \\ * Correspondence: anca.dinischiotu@bio.unibuc.ro \\ + Presented at the 2nd International Online-Conference on Nanomaterials, 15-30 November 2020; Available \\ online: https://iocn2020.sciforum.net/.
}

Published: 15 November 2020

\begin{abstract}
Due to their various optical and electronic features that offer advantages for medical purposes compared to traditional nanoparticles (NPs), quantum dots (QDs) represent an emerging tool for in vivo imaging, tumor biology investigation, and cancer treatment. Notwithstanding, QDs can also trigger toxicity effects in healthy cells, as previously reviewed by Zhu et al. [1]. Given that, we further aimed herein to characterize the silicon-based QDs obtained by laser ablation and evaluate their in vivo kidney toxicity. The studied NPs exhibited at transmission electronic microscope a core-shell structure with a crystalline silicon core and an amorphous silica shell with a diameter ranging between 6 and $10 \mathrm{~nm}$. Their tendency to aggregate led to the formation of aggregates with sizes of hundreds of nanometers. QDs dispersion in water revealed a hydrodynamic diameter around $200 \mathrm{~nm}$ and a negative zeta potential of $-14 \mathrm{mV}$. To test their in vivo toxicity, different doses of QDs (0,1 10 and $100 \mathrm{mg}$ QDs/kg body weight) prepared in $0.9 \%$ saline were injected in the caudal vein of the Swiss mice. The animals were sacrificed at 1, 6, 24 and $72 \mathrm{~h}$, and the kidney tissue was harvested. The effects of silicon QDs on the antioxidant defense of kidney cells were investigated throughout the assessment of antioxidant enzymes' activities (catalase, superoxide dismutase, glutathione peroxidase, glutathione reductase and glutathione S-transferase). The administration of the highest dose of QDs induced a significant reduction in catalase activity, the level being half of the control after all periods of exposure. A time-dependent decrease in glutathione reductase activity was noticed for all doses administered compared to control animals. After 24 and $72 \mathrm{~h}$, glutathione peroxidase and glutathione S-transferase were diminished in the kidney cells of mice that received 10 and $100 \mathrm{mg} / \mathrm{kg} \mathrm{b.w.} \mathrm{compared} \mathrm{to} \mathrm{control,}$ revealing that these enzymes were vulnerable to oxidative damage of high doses of silicon QDs. Yet, no significant changes were observed regarding the activity of superoxide dismutase in the kidney of treated mice compared to control, suggesting that the QDs administration would not generate superoxide anions inside kidney cells. This study highlighted the possible damaging effects of high doses of silicon-based QDs (>10 mg QDs/kg b.w.) on kidney cells, providing useful information for further clinical studies on humans.
\end{abstract}

Keywords: nanoparticles; quantum dots; toxicity

\section{Introduction}


Silicon-based Quantum Dots (Si QDs) are a special class of nanoparticles, due to the special properties they have: low toxicity and easily modifiable surface properties. For this reason, they are used in applications such as bioimaging, fluorescent labeling, drug administration, protein detection techniques, tissue engineering. In this context, we aimed to test the renal toxicity of silicone-based QDs obtained by laser ablation, in vivo.

\section{Materials and Methods}

In this study, adult male mice from the Swiss line were injected into the tail vein with different doses of QD (0, 110 , and $100 \mathrm{mg} \mathrm{QD} / \mathrm{kg}$ body weight) prepared in $0.9 \%$ saline. The injected nanoparticles were synthesized by the laser ablation method. After the expiration of the time periods after the injection of the nanoparticles $(1 \mathrm{~h}, 6 \mathrm{~h}, 24 \mathrm{~h}, 72 \mathrm{~h})$, the mice were sacrificed by cervical dislocation. The renal tissue was taken and cryogenized to be used later in the oxidative stress experiments (evaluating antioxidant enzymes: catalase, superoxide dismutase, glutathione peroxidase, glutathione reductase, and glutathione S-transferase).

\section{Results}

Evaluation of antioxidant enzyme activity (Figure 1) indicates a significant decrease in catalase levels at all periods of exposure. Both glutathione peroxidase and glutathione S-transferase exhibited a decrease in enzymatic activity at the highest concentrations (10 and $100 \mathrm{mg} / \mathrm{kg}$ ). Glutathione reductase activity decreased relative to the control unit per unit time after all periods of exposure. No significant changes were observed in superoxide dismutase activity.
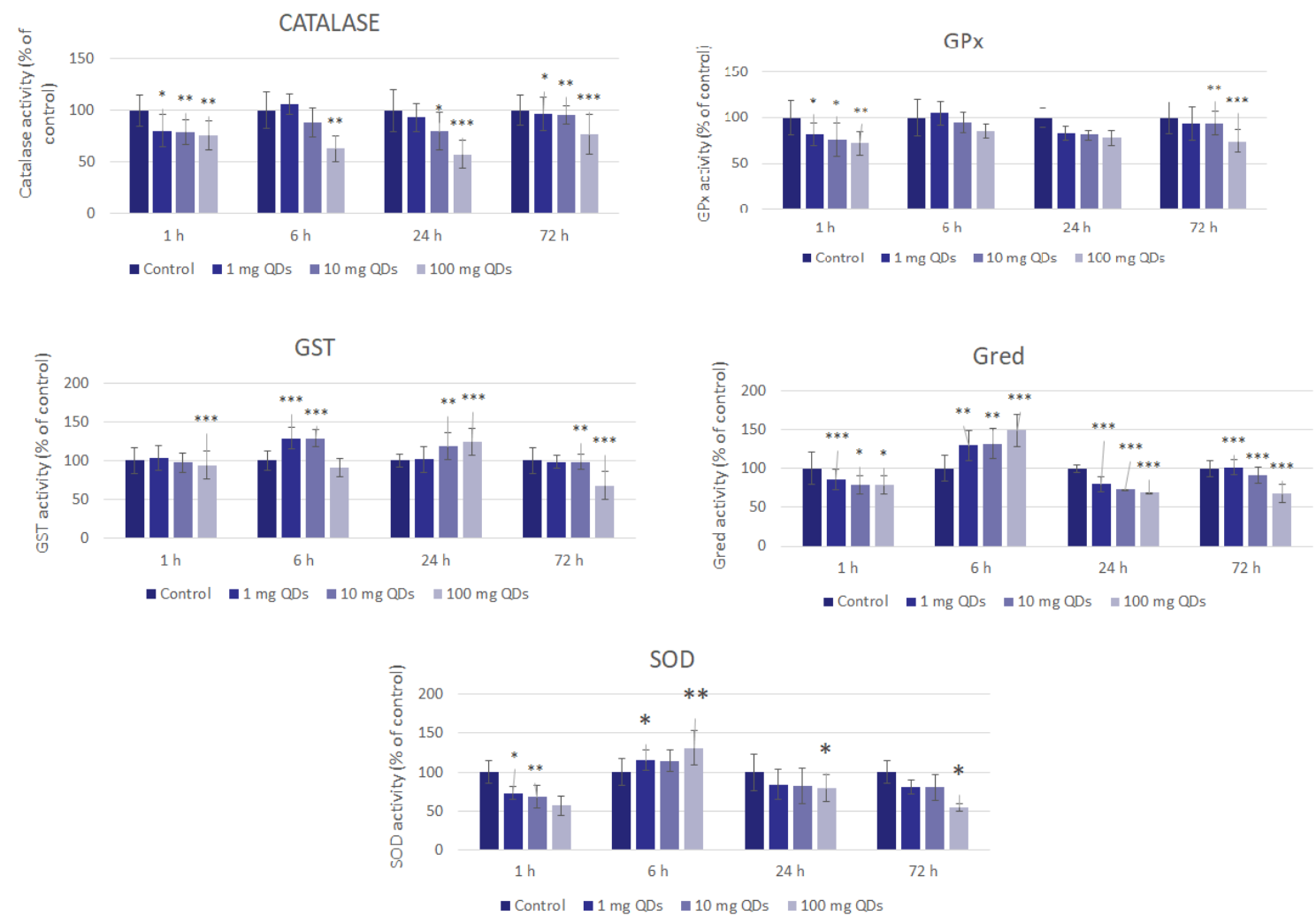

Figure 1. The evaluation of the activity of the antioxidant enzymes.

\section{Conclusions}

The experiments showed that nanoparticles of SiQDs synthesized by laser ablation could cause harmful effects on kidney tissue when administered in high doses (> $10 \mathrm{mg}$ QDs/kg body weight). Understanding how Si QDs nanoparticles act biologically and identifying the mechanisms by which 
they cause toxicity favors finding ways to design safer nanoparticles for the body and the environment.

Funding: M.S. Stan acknowledges the support of the Operational Programme Human Capital of the Ministry of European Funds through the Financial Agreement 51668/09.07.2019, SMIS code 124705.

Conflicts of Interest: The authors declare no conflict of interest. The funders had no role in the design of the study; in the collection, analyses, or interpretation of data; in the writing of the manuscript, or in the decision to publish the results.

Publisher's Note: MDPI stays neutral with regard to jurisdictional claims in published maps and institutional affiliations.

(c) 2020 by the authors. Licensee MDPI, Basel, Switzerland. This article is an open access article distributed under the terms and conditions of the Creative Commons Attribution (CC BY) license (http://creativecommons.org/licenses/by/4.0/). 